\title{
Endovascular intervention in elderly patients with peripheral arterial disease
}

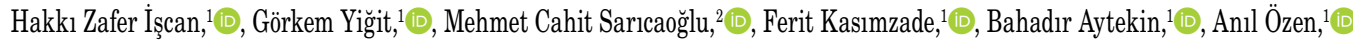 \\ ${ }^{1}$ Department of Cardiovascular Surgery, Ankara City Hospital, Ankara, Turkey \\ ${ }^{2}$ Department of Cardiovascular Surgery, Ankara University, Faculty of Medicine, Ankara, Turkey
}

\begin{abstract}
Objectives: In this study, we present our early and mid-term experiences following treatment of femoropopliteal lesions via percutaneous transluminal angioplasty (PTA) in patients over 70 years of age.

Patients and methods: This single-center, retrospective study included a total of 41 PTA procedures in 35 patients (23 males, 12 females; median age 74 years; range 70 to 87 years) due to femoropopliteal lesions between August 2015 and April 2018. The technical success rate was evaluated. The Kaplan-Meier analysis was used to evaluate the primary patency rate at 12 and 24 months.

Results: The mean follow-up was $14.9 \pm 8.9$ months. The technical success rate was $95.1 \%$. The bailout stenting was required in 12 patients (29.3\%). Only four patients (9.8\%) required percutaneous reintervention at the end of the follow-up. The Kaplan-Meier analysis revealed primary patency rates as $82.9 \%$ and $57.2 \%$ at 12 and 24 months, respectively.

Conclusion: Our study results support the idea that PTA in elderly patients with femoropopliteal lesions is a safe and effective option and can be applied with favorable results in the early and mid-term. In addition, endovascular procedures can be performed by cardiovascular surgeons with high success and low complication rates.
\end{abstract}

Keywords: Elderly, endovascular treatment, peripheral arterial disease.

The incidence of peripheral arterial disease (PAD) is 12 to $14 \%$ of the general population and increases up to $20 \%$ for patients older than 75 years of age. ${ }^{[1]}$ Previous studies have shown that revascularization procedures improve the quality of life and provide longer survival, compared to conservative treatment and primary amputation, in elderly patients. ${ }^{[2]}$

However, the benefits of revascularization may be limited due to the severity of comorbidities in the elderly. Such procedures are related to an increased peri- and postoperative mortality in this population. ${ }^{[3]}$

Early follow-up outcomes of endovascular procedures in the elderly provide better survival and patency rates, compared to surgery. ${ }^{[4]}$ Therefore, percutaneous transluminal angioplasty (PTA) may be considered as the primary treatment modality in these patients.
In this study, we present our early- and mid-term experiences following the treatment of femoropopliteal lesions performed by cardiovascular surgeons via PTA in the patients over 70 years of age.

\section{PATIENTS AND METHODS}

This single-center, retrospective study included a total of 41 PTA procedures in 35 patients $(23$ males, 12 females; median age 74 years; range 70 to 87 years) due to femoropopliteal lesions between August 2015 and April 2018. Patients who had atherosclerotic disease of femoral or popliteal arteries with symptoms of moderate intermittent claudication to diffuse pedal ischemia according to the Rutherford classification ${ }^{[5]}$ (Class 2 to 5) and who were not eligible for surgery due to comorbidities were included in the study. All patients in this study were admitted to the

Received: March 26, 2020 Accepted: April 21, 2020 Published online: June 16, 2020

Correspondence: Görkem Yiğit, MD. Altınkuleler Sitesi, Sakarya Mahallesi, Yönü Sokak, 06080 Altındağ, Ankara, Türkiye. e-mail: drgorkemyigit@gmail.com 
outpatient clinic with claudication symptoms despite the use of antilipidemic and antiaggregant agents and who underwent walking exercises within the past three months. The patients were physically fit and had relatively reasonable life expectancy with limited physical activity due to claudication. Patients with intermediate claudication and minor tissue loss were included. Those with major tissue loss and risk of amputation were excluded from the study. A written informed consent was obtained from each patient. The study protocol was approved by the Türkiye Yüksek İhtisas Training and Research Hospital, Ethics Committee (Date: 31.01.2017, No: 29620911-929). The study was conducted in accordance with the principles of the Declaration of Helsinki.

All clinical, perioperative, and demographic data were obtained through the review of original hospital and physician records, including data collected prospectively in the departmental registry. Demographic and clinical data including age, sex, hypertension, dyslipidemia, chronic obstructive pulmonary disease, and presence of coronary artery disease, chronic kidney disease, previous coronary artery bypass grafting, cigarette smoking, and previous percutaneous intervention were recorded.

\section{Operative technique}

All interventions were performed via PTA using the Luminor ${ }^{\circledR}$ paclitaxel-coated drug-eluting balloon catheter (iVascular, S.L.U., Barcelona, Spain) and bailout therapy with iVolution ${ }^{\circledR}$ selfexpanding nitinol stent (iVascular, S.L.U., Barcelona, Spain) for femoropopliteal lesions.

Prior to PTA, all patients were evaluated with Duplex ultrasound (DUS) and digital subtraction angiography (DSA). In our routine clinical practice, PTAs are performed under local anesthesia with monitorization by cardiovascular surgeons in the hybrid operating theatre. We usually prefer the antegrade femoral or contralateral retrograde femoral access. Following the insertion of a 7-Fr single lumen sheath, intravenous heparin is administered according to an activated clotting time of 180 to $200 \mathrm{sec}$. None of the lesions are predilated. All lesions are dilated with drug-coated balloons (DCBs) (at a vessel/balloon ratio of $1: 1$ on the basis of visual estimate) for a total inflation time of three min at 6 to $14 \mathrm{~atm}$. Balloons are inflated only once. However, in cases of flow-limiting dissection (i.e., interruption of the blood inflow due to a localized segment of dissection during DSA imaging) or residual stenosis (i.e., stenosis of the blood lumen greater than 50\%), bailout stenting is employed. When control angiography reveals a residual lesion $(>50 \%$ stenosis), flow-limiting dissection or plaque deformation, a second DCB procedure is carried out and dilatation is maintained for a longer period ( $\geq 3 \mathrm{~min}$ ).

After the procedure, the patients were scheduled for follow-up visits to the cardiovascular surgery outpatient clinics on a regular basis and were evaluated for patency of the vessels and restenosis using DUS and computed tomography angiography (CTA), when necessary. Post-interventional anklebrachial indices (ABIs) were also evaluated. The procedural technical success was defined as the achievement of a satisfactory patency of the vessel $(\geq 70)$ and an increased blood flow to the distal vessel at 12 and 24 months. The primary patency was defined as patency of the vessel using Doppler ultrasound (and CTA when necessary) and an increased blood flow to the distal vessel at 12 and 24 months. The Kaplan-Meier analysis was used to evaluate the primary patency rates during follow-up. A symptomatic stenosis greater than $50 \%$ of the vessel lumen was defined as residual stenosis.

\section{Statistical analysis}

Statistical analysis was performed using the SPSS version 16.0 software (SPSS Inc., Chicago, IL, USA). Descriptive data were expressed in mean \pm standard deviation (SD) or median (min-max) for continuous variables and in number and frequency for nominal variables. The Shapiro-Wilk test was used to check the normal distribution of continuous variables. Dependent intra-group variables were compared using the Wilcoxon test. A $p$ value of $<0.05$ was considered statistically significant.

\section{RESULTS}

A total of 35 septuagenarians and octogenarians were included in this study. Baseline demographic and clinical characteristics of the patients are shown in Table 1.

A DCB was used in 39 (95.1\%) of the lesions. The mean follow-up was $14.9 \pm 8.9$ (range, 6 to 24 ) months. The technical success rate was $95.1 \%$. Bailout stenting was required in 12 (29.3\%) of the lesions and only four patients $(9.8 \%)$ required percutaneous reintervention at the end of follow-up. Clopidogrel was prescribed to all patients; however, cilostazol was prescribed only for patients with a poor distal vasculature (Table 2). Blood glucose regulation was achieved in all patients. 
Table 1. Demographic and clinical data of patients undergoing $\mathrm{PTA} \pm$ bailout stenting

\begin{tabular}{lcccc}
\hline Demographics & $\mathrm{n}$ & $\%$ & Median & Range \\
\hline Age (year) & & & 74 & $70-87$ \\
Gender & & & & \\
$\quad$ Male & 23 & 65.7 & & \\
$\quad$ Female & 12 & 34.2 & & \\
Diabetes & 20 & 57.1 & & \\
Hypertension & 21 & 60 & & \\
Dyslipidemia & 10 & 28.5 & & \\
COPD & 7 & 20 & & \\
CAD & 14 & 40 & & \\
CKD & 3 & 8.5 & & \\
CABG & 5 & 14.2 & & \\
Smoking & 25 & 71.4 & & \\
Previous percutaneous intervention & 5 & 14.2 & &
\end{tabular}

PTA: Percutaneous transluminal angioplasty; COPD: Chronic obstructive pulmonary disease; CAD: Coronary artery disease; CKD: Chronic kidney disease; CABG: Coronary artery bypass grafting.

Table 2. Post-procedural data

\begin{tabular}{lccc}
\hline & $\mathrm{n}$ & $\%$ & $\mathrm{Mean} \pm \mathrm{SD}$ \\
\hline Clopidogrel & 41 & 100 & \\
Cilostazol & 18 & 43.9 & \\
Statin & 24 & 58.5 & \\
Hospitalization period (days) & & & $1.8 \pm 1.6$ \\
Claudication at control (Rutherford class $\geq 2)$ & 10 & 24.3 & \\
Follow-up period (months) & & & $14.9 \pm 8.9$ \\
Decision following check-up & & & \\
$\quad$ Medical follow-up & & & \\
$\quad$ Surgical intervention & 33 & 80.4 & \\
$\quad$ Percutaneous intervention & 4 & 9.7 & \\
\hline
\end{tabular}

SD: Standard deviation.

\section{Table 3. Lesion characteristics \& bailout stenting}

\begin{tabular}{lcc}
\hline & $\mathrm{n}$ & $\%$ \\
\hline Lesions & 41 & \\
Lesion length $(\mathrm{cm})$ & & \\
$\quad$ Short-medium $(\leq 15 \mathrm{~cm})$ & 32 & 78 \\
$\quad$ Long $(>15 \mathrm{~cm})$ & 9 & 21.9 \\
$\quad$ Infrapopliteal lesions & 23 & 56 \\
Bailout stenting & 12 & \\
Short-mean lesions & 8 & 25 \\
Long lesions & 4 & 44.4 \\
\hline
\end{tabular}

Although all patients were preoperatively symptomatic and had claudication, $75.6 \%$ of them had mild symptoms or were asymptomatic at the end of follow-up. The ABI was improved significantly after the procedure $(\mathrm{p}<0.001)$. Lesion characteristics

\section{Table 4. Procedural data}

\begin{tabular}{lccc}
\hline & $\mathrm{n}$ & $\%$ & Mean \pm SD \\
\hline Drug-coated balloon & 39 & 95.1 & \\
Stenting & 12 & 29.2 & \\
Technical success & 39 & 95.1 & \\
Complications & 6 & 14.6 & \\
Hematoma & 3 & 7.3 & \\
Pseudoaneurysm & 2 & 4.8 & \\
Infection & 1 & 2.4 & \\
Preprocedural ankle-brachial index & & & $0.4 \pm 0.1$ \\
Postprocedural ankle-brachial index & & & $0.8 \pm 0.2$ \\
Preprocedural creatinine level & & & $1.3 \pm 0.9$ \\
Postprocedural creatinine level & & & $1.3 \pm 1.1$ \\
\hline SD: Standard deviation. & & &
\end{tabular}

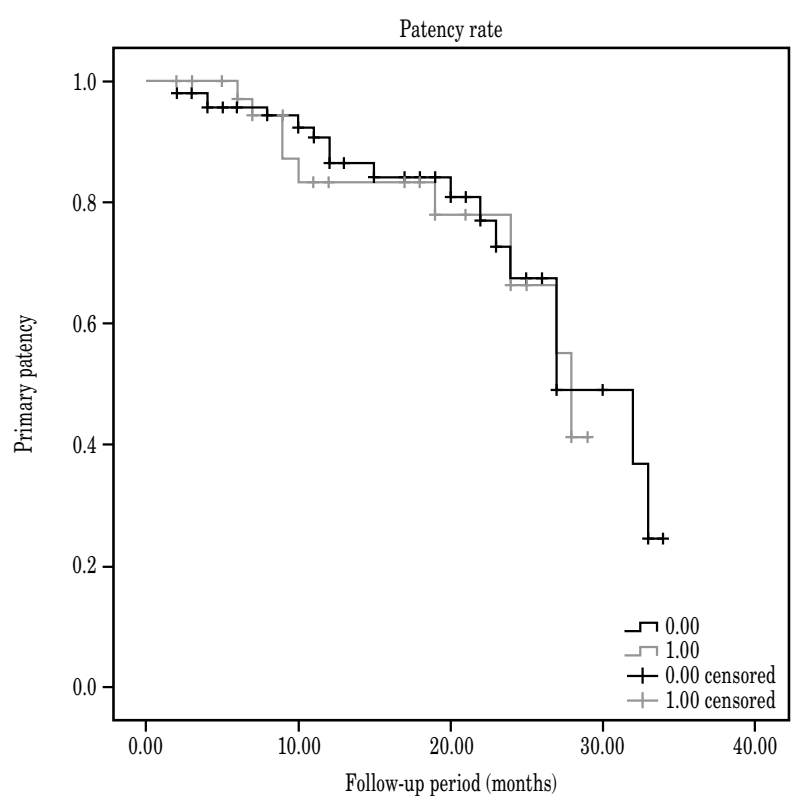

Figure 1. Kaplan-Meier analysis demonstrating primary patency rates at 12 and 24 months, respectively.

was classified as short-medium $(\leq 15 \mathrm{~cm})$ and long lesion $(>15 \mathrm{~cm})$. Eight of 32 patients $(25 \%)$ with short-intermediate lesions and four of nine patients (44.4\%) with long lesions underwent stenting (Table 3). Following endovascular interventions, three patients (8.5\%) developed hematomas, while two patients (5.7\%) developed infections and one patient $(2.8 \%)$ had a pseudoaneurysm of the femoral artery. Procedural details are shown in Table 4. During follow-up, none of the patients died or needed amputation. None of the patients withdrew their consents or were lost to follow-up, either. 
The primary patency rates as assessed by the Kaplan-Meier analysis were estimated as $82.9 \%$ and $57.2 \%$ at 12 and 24 months, respectively (Figure 1). Eight patients developed residual stenosis during follow-up, and four (12.5\%) underwent an endovascular and four (12.5\%) underwent an open surgical procedure.

\section{DISCUSSION}

In recent years, the number of elderly patients with PAD referred to the outpatient clinic has been on a rise due to the increased number of population and increased life expectancy. ${ }^{[6]}$ Most of the studies regarding endovascular intervention in elderly patients with PAD include patients with critical limb ischemia, whereas our study included elderly patients with chronical limb ischemia. In this study, we present our clinical experience in patients with chronic lower extremity ischemia aged 70 and over whose treatments were carried out by the cardiovascular surgeons.

In many centers, endovascular treatment is applied as the first-line therapy for the treatment of patients with leg ischemia. ${ }^{[7-9]}$ Previous studies have shown that, even in elderly patients, endovascular procedures are associated with improved outcomes. ${ }^{[2]}$ At the present day, even chronic total occlusions can be cured successfully using endovascular treatment. ${ }^{[10]}$ In our institution, performance of percutaneous revascularization procedures has developed over the past five years with primary surgical bypass devoted to patients whose percutaneous interventions have failed.

The first-line treatment in elderly patients includes risk modifications and medications. Therefore, the existing risk factors were also modified as the first step of the treatment in the patients in this study. These factors were smoking cessation, treatment of hypertension primarily with angiotensin-converting enzyme inhibitors, lowering of hemoglobin A1c levels below 7\%, anti-hyperlipidemic treatment primarily with statin, antiaggregant therapy primarily with clopidogrel, cilostazol therapy, and controlled exercise program. All patients in this study presented to the outpatient clinic with claudication symptoms, despite the use of antilipidemic and antiaggregant agents, and with walking exercises within the past three months. They were either not eligible for surgery due to comorbidities or refused open surgery.

Interventional or surgical revascularization, amputation in the presence of irreversible effects are the main treatment components. ${ }^{[11]}$ Nevertheless, the first choice of treatment modality has become endovascular therapy for elderly patients with PAD. Salas et al. ${ }^{[12]}$ showed the 24-month primary patency rate as 52\%. In addition, Dosluoglu et al. ${ }^{[4]}$ reported a 12 -month primary patency rate of $78 \%$ for endovascular-treated elderly patients. Compared to the aforementioned studies, the patency rates were $82.9 \%$ and $57.2 \%$ at 12 and 24 months, respectively in our study, indicating higher patency rates. However, the number of patients in these studies were relatively large ( $n=98$ and $n=344$, respectively).

Regardless of the treatment modality, elderly patients with leg ischemia have an annual mortality rate of 29\%.. ${ }^{[13]}$ Furthermore, the perioperative mortality rates in series of elderly patients with lower limb ischemia varies from 2 to $12 \%$ following endovascular revascularization. ${ }^{[4,14-16]}$ In a prospective cohort study, Brosi et al. ${ }^{[2]}$ reported a 30 -day mortality of $6 \%$ after endovascular treatment, $20 \%$ after surgical revascularization, and $17.9 \%$ after conservative treatment in octogenarians. Aforementioned studies reveal a lower mortality rate for endovascular procedures compared to surgery, and the peri- and postoperative mortality rate during a two-year follow-up was $0 \%$ in our study. This is probably due to the small number of patients in our study and the lack of other series including complications such as poor medical condition, cardiovascular morbidity, or high infection rates. However, our study involved 14 patients with a history of coronary artery disease, five patients with a previous coronary artery bypass grafting, and four patients with renal insufficiency.

The extremity recovery and symptomatic recovery rates were also excellent in our study, indicating satisfactory outcomes. It is difficult to predict adverse postoperative outcomes, particularly in elderly patients due to severe comorbidities. Thus, choosing the most optimal treatment method for each individual patient is critical for surgeons. A recent, randomizedcontrolled study of Mietz et al. ${ }^{[17]}$ revealed that application of DCBs for femoropopliteal lesions resulted in a decrease in restenosis and target vessel revascularization at 12 months. As many studies and the present study suggest, endovascular procedures are associated with fewer complication rates and improved vessel patency rates.

Our study has some limitations due to its retrospective design, small number of patients, and relatively short follow-up period.

In conclusion, our study results support the idea that PTA in the elderly patients with femoropopliteal 
lesions is a safe and effective option and can be applied with favorable results in the early- and mid-term. In addition, PTA is a less invasive alternative in elderly patients with chronic limb ischemia and cardiovascular surgeons should be encouraged to perform PTA in this patient population with high success and low complication rates.

\section{Declaration of conflicting interests}

The authors declared no conflicts of interest with respect to the authorship and/or publication of this article.

\section{Funding}

The authors received no financial support for the research and/or authorship of this article.

\section{REFERENCES}

1. Shammas NW. Epidemiology, classification, and modifiable risk factors of peripheral arterial disease. Vasc Health Risk Manag 2007;3:229-34.

2. Brosi P, Dick F, Do DD, Schmidli J, Baumgartner I, Diehm N. Revascularization for chronic critical lower limb ischemia in octogenarians is worthwhile. J Vasc Surg 2007;46:1198-207.

3. Plecha FR, Bertin VJ, Plecha EJ, Avellone JC, Farrell CJ, Hertzer NR, et al. The early results of vascular surgery in patients 75 years of age and older: an analysis of 3259 cases. J Vasc Surg 1985;2:769-74.

4. Dosluoglu HH, Lall P, Cherr GS, Harris LM, Dryjski ML. Superior limb salvage with endovascular therapy in octogenarians with critical limb ischemia. J Vasc Surg 2009;50:305-15, 316.e1-2.

5. Rutherford RB, Baker JD, Ernst C, Johnston KW, Porter JM, Ahn S, et al. Recommended standards for reports dealing with lower extremity ischemia: revised version. J Vasc Surg 1997;26:517-38.

6. Diehm C, Schuster A, Allenberg JR, Darius H, Haberl $\mathrm{R}$, Lange $\mathrm{S}$, et al. High prevalence of peripheral arterial disease and co-morbidity in 6880 primary care patients: cross-sectional study. Atherosclerosis 2004;172:95-105.

7. Aydin S. Combined use of atherectomy and drug-coated balloon for endovascular treatment of femoropopliteal artery disease. Turk J Vasc Surg 2019;28:84-90.

8. Black JH 3rd, LaMuraglia GM, Kwolek CJ, Brewster DC, Watkins MT, Cambria RP. Contemporary results of angioplasty-based infrainguinal percutaneous interventions. J Vasc Surg 2005;42:932-9.

9. DeRubertis BG, Faries PL, McKinsey JF, Chaer RA, Pierce M, Karwowski J, et al. Shifting paradigms in the treatment of lower extremity vascular disease: a report of 1000 percutaneous interventions. Ann Surg 2007;246:415-22.

10. Yiğit G, Cahit Sarıcaoğlu M, Çetinkaya F, Özen A, Zafer İşcan H. Endovascular treatment of chronic total occlusion of iliac/femoral arteries: Mid-term follow-up. Turk J Vasc Surg 2020;29:007-12.

11. Balkanay OO, Ömeroğlu SN. Approach to peripheral arterial disease in the elderly. Turk Kardiyol Dern Ars 2017;45:96-101.

12. Salas CA, Adam DJ, Papavassiliou VG, London NJ. Percutaneous transluminal angioplasty for critical limb ischaemia in octogenarians and nonagenarians. Eur J Vasc Endovasc Surg 2004;28:142-5.

13. Norgren L, Hiatt WR, Dormandy JA, Nehler MR, Harris $\mathrm{KA}$, Fowkes FG, et al. Inter-Society Consensus for the Management of Peripheral Arterial Disease (TASC II). Eur J Vasc Endovasc Surg 2007;33:S1-75.

14. Pomposelli FB Jr, Arora S, Gibbons GW, Frykberg R, Smakowski P, Campbell DR, et al. Lower extremity arterial reconstruction in the very elderly: successful outcome preserves not only the limb but also residential status and ambulatory function. J Vasc Surg 1998;28:215-25.

15. Arvela E, Venermo M, Söderström M, Korhonen M, Halmesmäki K, Albäck A, et al. Infrainguinal percutaneous transluminal angioplasty or bypass surgery in patients aged 80 years and older with critical leg ischaemia. Br J Surg 2011;98:518-26.

16. Scher LA, Veith FJ, Ascer E, White RA, Samson RH, Sprayregen $\mathrm{S}$, et al. Limb salvage in octogenarians and nonagenarians. Surgery 1986;99:160-5.

17. Mietz S, Lehmann T, Teichgräber U. Multivariable Regression Analysis of Clinical Data from the RandomizedControlled EffPac Trial: Efficacy of Femoropopliteal Drug-Coated Balloon Angioplasty. Cardiovasc Intervent Radiol 2020;43:840-9. 